\title{
PROBLEM OF COUPLED THERMOELASTICITY FOR A HALF-LAYER WITH A TUNNEL CAVITY: AN ANTISYMMETRIC CASE
}

\author{
L. A. Fil'shtinskii" and A. V. Bondar'
}

\begin{abstract}
A boundary-value problem of coupled thermoelasticity for a half-layer with a hole and mixed boundary conditions is solved. The problem is reduced to a system of four singular integral equations. It is solved numerically using the mechanical-quadrature method. A numerical experiment is conducted to study the dynamic stress concentration around cavities of different cross sections. The effect of the coupled thermal and elastic fields on the wave processes in the body is established
\end{abstract}

Keywords: coupled dynamic thermoelasticity, half-layer, tunnel cavity, mixed boundary conditions, singular integral equations of the second kind, dynamic stress concentration

Introduction. Modern mechanical engineering widely uses materials with strong coupling of the strain and thermal fields. Such materials, used to manufacture insulating coatings, building structures, unbreakable glass, etc., include aldehydic materials such as polyvinyl formal, polyvinyl butyral, polystyrene, etc. Many structures are subjected to intensive dynamic mechanical and thermal loads; therefore, there is a need to develop methods to analyze coupled thermal and elastic fields.

Problems of coupled thermoelasticity are solved mainly by two methods. One method eliminates time from partial differential equations by using the Laplace transform $[5,6]$, while the other method eliminates the space coordinates by expanding the solution into an appropriate orthonormal series or using a finite (infinite) integral transform in the spatial domain $[7,11]$. Since the use of these methods involves severe mathematical difficulties, asymptotic methods have become popular. Most studies on the subject are concerned with problems for a thermoelastic half-space under mechanical loads uniformly distributed over the surface $[16,18,19]$. Dynamic coupled problems for a flat layer, an infinite cylinder, and a sphere are addressed in $[2,5,6,8,10,11,20,23]$. The generalized coupled problem of thermoelasticity for a space with a spherical cavity is solved in [24]. Dynamic problems of thermoelasticity for thin-walled structural members under mechanical and thermal loads are solved in [1, 4]. The heat conductivity and thermoelastic deformation of shells and solids of revolution subject to local or impulsive heating are studied in [15, 21, 22]. The coupling of the thermal and elastic fields in a layer and a cylinder of finite length under dynamic loads is examined in $[12,13,17]$.

Considering the foregoing, one of the authors has developed a new approach to solve three-dimensional problems of elasticity for multiply connected plates and cylindrical shells. The approach employs F-solutions for a layer and reduces problems to one-dimensional integral equations, which are well understood. The method of F-solutions is extended below to the dynamic coupled problem of thermoelasticity for a half-layer weakened by a hole along the $x_{3}$-axis.

1. Basic Equations and F-Solutions for a Layer and a Half-Layer. After the elimination of the time factor $e^{-i \omega t}$, the equations of coupled thermoelasticity take the following form [2]:

$$
\begin{gathered}
\Delta U_{j}+\sigma \partial_{j} e-\frac{3 \lambda+2 \mu}{\mu} \alpha_{T} \partial_{j} U_{4}+\gamma_{2}^{2} U_{j}+\frac{1}{\mu} X_{j}^{*}=0 \quad(j=1,2,3), \\
\Delta U_{4}+\left(\gamma_{T}^{2}+i \gamma^{2}\right) U_{4}+i m \omega e+\frac{Q^{*}}{\lambda_{T}}=0
\end{gathered}
$$

Sumy State University, 2 Rimsky-Korsakov St., Sumy, Ukraine 40007, e-mail: leonid@mphis.sumdu.edu.ua. Translated from Prikladnaya Mekhanika, Vol. 44, No. 10, pp. 28-36, October 2008. Original article submitted April 3, 2007. 